\title{
Cardinal Motor Features of Parkinson's Disease Coexist with Peak-Dose Choreic-Type Drug-Induced Dyskinesia
}

\author{
Etienne Goubault $^{\mathrm{a}, \mathrm{b}}$, Hung P. Nguyen ${ }^{\mathrm{a}, \mathrm{b}}$, Sarah Bogard ${ }^{\mathrm{a}, \mathrm{b}}$, Pierre J. Blanchet ${ }^{\mathrm{c}, \mathrm{d}}$, Erwan Bézard ${ }^{\mathrm{e}, \mathrm{f}}$, \\ Claude Vincent ${ }^{\mathrm{g}}$, Mélanie Langlois ${ }^{\mathrm{h}}$ and Christian Duval ${ }^{\mathrm{a}, \mathrm{b}, *}$ \\ ${ }^{a}$ Département des Sciences de l'activité physique, Université du Québec à Montréal, Montréal, Québec, Canada \\ ${ }^{\mathrm{b}}$ Centre de Recherche de l'Institut universitaire de gériatrie de Montréal, Montréal, Québec, Canada \\ ${ }^{\mathrm{c}}$ Département de stomatologie, Faculté de médecine dentaire, Université de Montréal, Montréal, QC, Canada \\ ${ }^{\mathrm{d}}$ Département de médecine, CHU Montréal, Montréal, QC, Canada \\ ${ }^{\mathrm{e}}$ Université de Bordeaux, Institut des Maladies Neurodégénératives, F-33000 Bordeaux, France \\ ${ }^{\mathrm{f}}$ Centre National de la Recherche Scientifique Unité Mixte de Recherche 5293, Institut des Maladies \\ Neurodégénératives, F-33000 Bordeaux, France \\ ${ }^{\mathrm{g}}$ Département de réadaptation, Faculté de médecine, Université Laval, Québec, Québec, Canada \\ ${ }^{\mathrm{h}}$ Département de médecine, Faculté de médecine, Université Laval, Québec, Québec, Canada
}

\begin{abstract}
.
Background: Clinical and anecdotal observations propose that patients with Parkinson's disease (PD) may show druginduced dyskinesia (DID) concomitantly with cardinal motor features. However, the extent of the concomitant presence of DID and cardinal features remains to be determined.

Objectives: This cross-sectional study measured peak-dose choreic-type DID in a quantitative manner in patients diagnosed with PD, and determined whether symptoms such as tremor, bradykinesia, rigidity, postural instability or freezing of gait (FoG) were still detectable in these patients.

Methods: 89 patients diagnosed with PD were recruited and assessed using a combination of quantitative measures using inertial measurement units to capture DID, tremor, bradykinesia, and FoG. Clinical evaluations were also used to assess rigidity and postural instability. Motor symptoms of PD were assessed 3 times during the testing period, and a series of activities of daily living were repeated twice, in between clinical tests, during which the level of DID was quantified. Peakdose was identified as the period during which patients had the highest levels of DID. Levels of tremor, rigidity, bradykinesia, postural instability, and FoG were used to determine the percentage of patients showing these motor symptoms simultaneously with DID.
\end{abstract}

\footnotetext{
*Correspondence to: Christian Duval, PhD, Département des Sciences de l'activité physique, Université du Québec à Montréal, C.P. 8888, succursale Centre-Ville, Montréal QC, H3C 3P8,
}

Canada. Tel.: +1 514987 3000/Ext. 4440; Fax: +1 514987 6616; E-mail: duval.christian@uqam.ca. 
Results: $72.4 \%$ of patients tested presented with measurable DID during the experiment. Rest, postural and kinetic tremor $(12.7 \%, 38.1 \%$, and $15.9 \%$ respectively), bradykinesia (28.6\%), rigidity (55.6\%), postural instability (71.4\%) and FoG $(9.5 \%)$ were detected simultaneously with DID.

Conclusions: PD symptomatology remains present in patients showing peak-dose choreic-type DID, illustrating the challenge facing physicians when trying to avoid dyskinesia while attempting to alleviate motor symptoms.

Keywords: Chorea, drug-induced, dyskinesia, hypokinesia, movement disorders, Parkinson disease, tremor

\section{ABBREVIATIONS}

$\begin{array}{ll}\text { ADL } & \text { Activities of daily living } \\ \text { DID } & \text { Drug-induced dyskinesia } \\ \text { FoG } & \text { Freezing of gait } \\ \text { PD } & \text { Parkinson's disease } \\ \text { RAM } & \text { Rapid alternating movement } \\ \text { TUG } & \text { Timed-up and go }\end{array}$

\section{INTRODUCTION}

Cardinal motor features of Parkinson's disease (PD), including bradykinesia, rigidity, tremor and postural instability $[1,2]$, are managed with pharmacological therapy $[3,4]$. Long-term use of those agents [3], combined with the further progressing loss of neurons producing dopamine [5], will eventually lead to pre- and post-synaptic plasticity [6-9] creating motor complications such as drug-induced dyskinesia (DID). Treating DID usually consists in reducing levodopa dosage, and/or adding new drugs $[4,10]$. However, as the disease progresses, this becomes increasingly challenging due to the narrowing of levodopa therapeutic window, as illustrated in Fig. 1 [11, 12].

Early in the disease, patients have a good response to the medication, allowing them to be in an ON state without fluctuating in the OFF state. However, previous research already suggested that anti-parkinsonian medication does not render patients 'normal', as they can still exhibit bradykinesia [13], tremor [14] or postural instability [15] during $O N$ periods. Later in the disease, there seems to be a closing of the therapeutic window where the $O N$ state can be overshot for an $O N$ state with dyskinesia. Clinical and anecdotal observations seem to suggest that advanced patients having DID can also present with cardinal features of PD [16-19]. However, what is not known is the extent of that concomitant presence of DID and cardinal motor features since no studies have addressed specifically this issue. If reducing drug dosage to manage DID is to be considered as a strategy to alleviate DID, it becomes important to determine which symptoms of

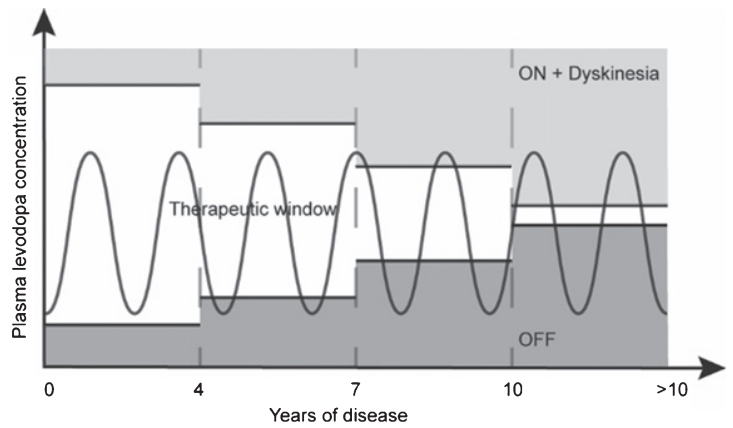

Fig. 1. Pattern of motor response to levodopa during the progression of PD. Early in the disease, levodopa response is optimal, reaching the therapeutic window. As the disease progresses, this therapeutic window decreases due to changes in exogenous dopamine management by remaining neurons. After approximately seven to ten years, this therapeutic window becomes less attainable, leaving the patient in either an $O F F$ or $O N$ with dyskinesia condition. Inspired by Cenci [11] and Jankovic [12].

PD may already be present in these patients since they may be exacerbated by this management strategy. Freezing of gait ( $\mathrm{FoG})$ was also considered in this study since it is known to be associated with higher risks of falls [20], and it is less sensitive to exogenous dopaminergic therapy. Therefore, the aims of this study were to measure the presence of peak-dose choreic-type DID in an objective manner, and determine the proportion of tremor, bradykinesia, rigidity, postural instability, or FoG present in these patients.

\section{METHODS}

\section{Research design}

A cross-sectional design with a control group was used for the study. Data were collected in two clinical research laboratories: the Centre de Recherche de l'Institut universitaire de gériatrie de Montréal (CRIUGM), and the Institut de Réadaptation en Déficience Physique de Quebec (IRDPQ), from June 2016 until November 2016. 
Standard protocol approvals, registrations, and patient consents

The study protocol was approved by each institutional research ethics review board (CER IUGM 13-14-022; MP-2016-510) and each participant read and signed an informed consent form.

\section{Participants}

Eighty-nine patients (thirty-five females) diagnosed with idiopathic PD according to the UK Parkinson's Disease Society Brain Bank clinical diagnosis criteria [21] were recruited with the help of the Quebec Parkinson Network (QPN), which is composed of clinicians specialized in movement disorders. Patients were selected if they demonstrated clinically-detectable choreic-type DID in the past months prior to testing. Patients requiring assistance to walk, or having orthopedic conditions, judged by our investigator to interfere with the required tasks, were not considered for the study. Thirtytwo healthy participants of comparable age (sixteen females, $65.7 \pm 8.6$ years old, MMSE mean score of $29.1 \pm 1.2$, GDS-15 mean score of $1.5 \pm 2.0$ ) were recruited through the Centre de Recherche de l'Institut universitaire de gériatrie de Montréal (CRIUGM), to establish a 'normal' baseline of behavior.

\section{Standard protocol or procedures}

Each visit to the laboratory was planned to coincide with their scheduled medication dose deemed to generate the highest DID amplitude. For most patients, this occurred in the afternoon. Upon arrival at the laboratory, they were instructed to take their medication, just prior to proceeding with the administrative requirements for the study participation, i.e., reading and signing the informed consent form.

For this study, we favored a quantitative approach to assess the levels of DID, tremor, bradykinesia, and FoG. To do so, participants were equipped with a motion capture suit (Animazoo IGS-180, Synertial UK Ltd) with 17 inertial measurement units, positioned on each body limb, each containing a 3-axis accelerometer, a 3-axis gyroscope, and a 3-axis magnetometer. We previously tested the accuracy of these sensors [22, 23], developed automated quality control [24] and segmentation algorithms to assess the mobility of elderly [25-27] and patients with PD [28, 29].
The protocol was initiated as soon as the investigators visually noticed choreic-type DID. The testing period proceeded in two distinct blocks of tasks, during which investigators asked patients for feedback about the amplitude of DID to ensure that they were at their highest level during the experiment. The first block was used for the assessment of cardinal motor features of PD: (a) Bradykinesia was assessed using a rapid alternating movement (RAM) task [30-32], described elsewhere [13, 33-35]. In brief, averaged maximal angular velocity of each full cycle of pronation-supination during fast repetitive pronation-supination movements, performed during 10 seconds, was used as the outcome measure of bradykinesia [13, 31, 33]; (b) Postural tremor was assessed while participants were standing, with arms stretched horizontally in front of them, for 20 seconds. Signals from gyroscopes $(\mathrm{x}, \mathrm{y}, \mathrm{z})$ positioned on the hands were band-pass filtered between 2 and $8 \mathrm{~Hz}$ to remove noise from the signal, and then detrended. The power spectral density was calculated for $\mathrm{x}, \mathrm{y}$, and $\mathrm{z}$ directions. These components were summed to generate the total power spectral density. If the dominant frequency of this resulting spectrum was between $3.5-7.5 \mathrm{~Hz}$ bandwidth, peak power (defined as the power estimation around the dominant frequency with $\pm 0.5 \mathrm{~Hz}$ width) was calculated. If the dominant frequency was out of the usual PD tremor frequency band, tremor amplitude was considered irrelevant (i.e., within the physiological range), and the amplitude was set to zero; (c) Postural instability and rigidity were both assessed using the Movement Disorder Society-Unified Parkinson's Disease Rating Scale (MDS-UPDRS, items 3.12 and 3.3, respectively), since robust objective assessment of these symptoms is not yet available. This block 1 was repeated three times.

During a second block, which was repeated twice (in between blocks 1), participants performed a series of activities of daily living (ADL) composed of reading, counting money, cutting and eating a piece of apple, eating soup, taking medication (candy), and drinking water.

The second block of tasks allowed us to capture DID levels during movement, as it is known to increase during voluntary motor behavior $[13,36]$. Once cued by a light situated in front of them, patients were instructed to perform an ADL at their preferred pace to reproduce true living conditions. Rest tremor was assessed just prior to movement when patients had their hands resting on the table. Kinetic tremor was assessed during the performance of these ADL 
using the same signal analysis approach that was used to evaluate postural tremor. In order to ensure that the tremor measured was indeed present during movement, the peak power associated with tremor had to be accompanied with significant power below $3 \mathrm{~Hz}$ associated with the voluntary movement.

Participants' DID, or lack of, was recorded at multiple points during the testing protocol where participants were either at rest or performing ADL. DID was quantified using all sensors, except those positioned on the arms involved in voluntary movements. Signals from gyroscopes $(\mathrm{x}, \mathrm{y}, \mathrm{z})$ were band-pass filtered between 0.5 and $10 \mathrm{~Hz}$ to remove noise from the signal, and then detrended. The magnitudes of the coordinate vectors $(\mathrm{x}, \mathrm{y}, \mathrm{z})$ were computed at each frame resulting in new time series which were then segmented into five-second windows. Power spectral densities were computed on each window and the energy spectrums were calculated. The sum of energies was then calculated and divided by the length of the window, given an average energy value per second, for each window of each task. We used the maximum energy as the outcome measure of DID amplitude.

In this block, participants also performed several walking tasks consisting of three different Timed-up and go (TUG) tasks ( 3 meters TUG; 3 meters TUG while carrying a glass of water; 3 meters TUG with an obstacle to step over), allowing us to assess freezing of gait (FoG) using an algorithm proposed by Moore et al. [37]. In brief, FoG results from an absence of forward locomotion and high frequency trembling of the lower limbs occurring in the $3-8 \mathrm{~Hz}$ bandwidth, whereas walking is characterized by a power spectrum below $3 \mathrm{~Hz}$. Therefore, a ratio between the power of the freezing bandwidth and the walking bandwidth is used on linear acceleration of multiple sensors (feet, shanks, thighs, and hip) to determine when participants are in a frozen state. To be in a frozen state, this ratio should be above the threshold determined by Moore et al. [37] for at least four of the seven sensors used in this algorithm. The percent time frozen during walking was calculated for each TUG task. The mean of each TUG task's percent time frozen was used as the outcome measure for FoG.

The order of tasks within Block 1 (symptomatology assessment) remained the same but was randomized for Block 2 (ADL). The order of block presentation remained the same for all participants, i.e., Block 1 - Block 2 - Block 1- Block 2 - Block 1. To ensure that we assessed the symptomatology of patients when they presented with clear peak-dose choreic-type DID, we first identified the period during which the amplitude of DID was the highest. Then, we used outcome measures such as the levels of DID amplitude, bradykinesia, rigidity, postural instability, FoG and tremor measurements that were closest in time (i.e., \pm 10 minutes) from that peak. Patients were in our laboratory for approximately 2 hours and the entire recording session lasted generally 1 hour. This period allowed us to ensure that patients having DID were experiencing choreic-type DID happening usually during one period, when patients are at peak-dose $[19,38]$.

\section{Statistics}

To determine the percentage of patients showing cardinal motor symptoms simultaneously with DID, we first established a 'normal' range of values from the control group. For clinically assessed symptoms, i.e., rigidity and postural instability, control values were zero, as expected. For quantifiable symptoms, i.e., DID, tremor, bradykinesia and FoG, the mean of healthy controls data plus two standard deviations was calculated and defined as thresholds. The percentage of patients having peak-dose DID could then be calculated, as well as whether patients presented other symptoms within the same period.

\section{RESULTS}

From the 89 participants with PD, two were excluded from the analysis; one presented with severe osteoarthritis, the other did not complete the experiment because of severe postural instability. From the 87 remaining patients tested, 63 presented with measurable DID that reached our threshold during the testing period, representing $72.4 \%$ of all patients tested (Fig. 2). The characteristics of these patients are presented in Table 1.

Figure 3 illustrates that in those 63 patients having DID, $28.6 \%$ presented with bradykinesia, $12.7 \%$ with rest tremor, $38.1 \%$ with postural tremor, $15.9 \%$ with kinetic tremor, $55.6 \%$ with rigidity, $71.4 \%$ with postural instability, and $9.5 \%$ with FoG concomitantly with DID.

The results also revealed that $96.8 \%$ of patients having DID presented at least one of these motor symptoms. More precisely, $20.6 \%$ presented only one other motor symptom, $38.1 \%$ presented two other symptoms, $22.2 \%$ presented three other symptoms, $11.1 \%$ presented four other symptoms, and $4.8 \%$ presented five other symptoms concomitantly with DID. 


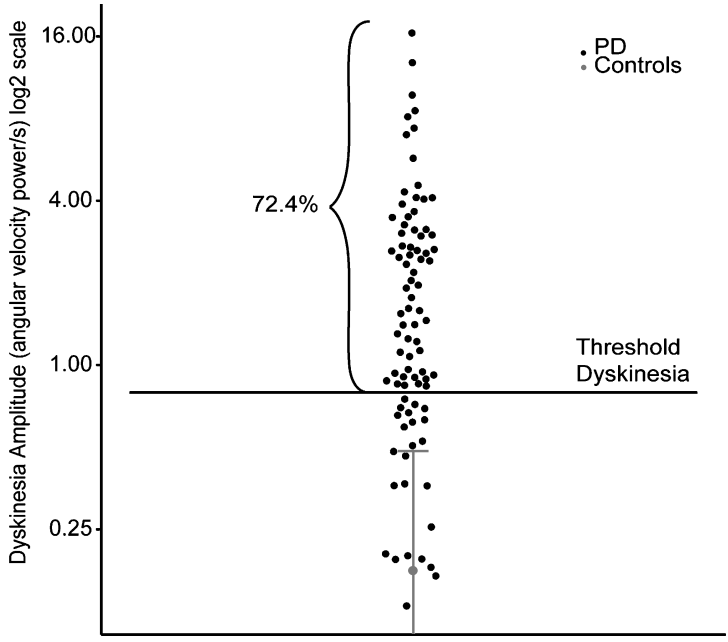

Fig. 2. Dyskinesia amplitude of controls and participants with PD. Dyskinesia detection in patients (black) based on the behavior of healthy controls (grey). Mean plus two standard deviations of data from controls are illustrated in gray. Individual data points from patients are illustrated in black.

Table 1

Characteristics of participants having DID

\begin{tabular}{|c|c|c|}
\hline Characteristics & Mean \pm SD & Range \\
\hline Age (y): min/max; mean \pm SD & $67.1 \pm 8.0$ & $49-83$ \\
\hline MMSE (/30) & $27.1 \pm 2.4$ & $20-30$ \\
\hline GDS-15 $(/ 15)^{\mathrm{b}}:$ & $3.9 \pm 2.7$ & $0-10$ \\
\hline Years since diagnosis & $10.1 \pm 4.7$ & $3-26$ \\
\hline $\operatorname{LEDD~}(\mathrm{mg})^{\mathrm{a}}$ & $977.7 \pm 540.1$ & $6-2790$ \\
\hline \multicolumn{3}{|l|}{ MDS-UPDRS part III ON ${ }^{b}$} \\
\hline Speech & $1.3 \pm 1.1$ & $0-4$ \\
\hline Facial expression (3.2) & $1.5 \pm 1.0$ & $0-4$ \\
\hline Arms rigidity $(3.3)^{\mathrm{c}}$ & $0.6 \pm 0.6$ & $0-2.5$ \\
\hline Legs rigidity $(3.3)^{\mathrm{c}}$ & $1.0 \pm 0.6$ & $0-2.5$ \\
\hline Arising from chair (3.9) & $0.4 \pm 0.7$ & $0-3$ \\
\hline Gait (3.10) & $1.1 \pm 0.9$ & $0-3$ \\
\hline Freezing of gait (3.11) & $0.3 \pm 0.7$ & $0-4$ \\
\hline Postural stability (3.12) & $1.1 \pm 0.9$ & $0-4$ \\
\hline Posture (3.13) & $0.7 \pm 0.9$ & $0-3$ \\
\hline Bradykinesia (3.14) & $1.2 \pm 1.1$ & $0-4$ \\
\hline Postural tremor $(3.15)^{\mathrm{c}}$ & $0.5 \pm 0.9$ & $0-4$ \\
\hline Rest tremor $(3.17)^{\mathrm{c}}$ & $0.3 \pm 0.5$ & $0-3$ \\
\hline \multicolumn{3}{|l|}{ MDS-UPDRS part IV ${ }^{\mathrm{b}}$ : } \\
\hline Time spent with dyskinesia (4.1) & $1.3 \pm 0.7$ & $0-4$ \\
\hline Functional impact of dyskinesia (4.2) & $1.4 \pm 0.9$ & $0-4$ \\
\hline Hoehn and Yahr score $\mathrm{ON}^{\mathrm{b}}$ & $2.5 \pm 1.0$ & $1-4$ \\
\hline
\end{tabular}

${ }^{\mathrm{a}}$ Missing data for 11 participants.; ${ }^{\mathrm{b}}$ Higher score indicates worse functioning.; ${ }^{\mathrm{c}}$ Score represents the mean of the left and right segments.; MMSE, Mini-Mental State Evaluation; GDS-15, 15-item Geriatric Depression Scale; MDS-UPDRS, Movement Disorder Society-Unified Parkinson's Disease Rating Scale; SD, standard deviation, LEDD, Levodopa Equivalent Daily Dose.

\section{DISCUSSION}

The novelty of the present study is that we quantitatively demonstrated that a significant portion of patients who presented with peak-dose choreic-type DID also showed cardinal motor features normally associated with PD; a concept only qualitatively alluded by others [16-19]. These results support our previous observations that bradykinesia, for instance, can be observed with DID [13, 39]. It is important to note that a significant portion of patients tested did not present with overwhelming DID, as the great majority of them were well-managed. In fact, DID was not consistently present at rest in a good proportion of patients. It is quite possible that exposing patients to higher doses of anti-parkinsonian drugs could have enhanced DID and further reduce any underlying PD symptoms. However, we think that testing patients in their real-life condition or state highlights the symptomatology they live with every day, reinforcing the impact of this study.

The physiological basis of the concomitant presence of DID and PD symptomatology could be explained by the fact that each symptom does not respond the same way to exogenous dopamine, suggesting the implication of different neuropathophysiological circuits. For DID, it is proposed that it is the results of a combination of factors which include hypersensitivity of post-synaptic receptors [9], loss of pre-synaptic neurons managing dopamine release [8] and altered transmitter release from neurons normally not associated with dopamine release $[8,40]$. Specifically, altered sensitivity of GABAergic medium spiny neurons that express predominantly D1 receptors associated with the direct pathway may play a part in DID [9]. If true, it is possible that hyperactivity of D1-modulated pathways generates DID, while D2-modulated pathways and other systems retain their 'parkinsonian' behavior. Then, dysfunction of basal ganglia output structures [41] to the thalamus and cortex could still induce bradykinesia [42]. Here, we gave a special attention to the $28 \%$ of patients having bradykinesia concomitantly with DID, to observe that their clinical characteristics such as age and medication levels were similar to the others. For rigidity, altered connectivity in subcortical and cortical regions, as well as the long loop and spinal reflex pathways, may play an important role [43-45]. In our group of patients, the great majority of them presented with rigidity that was detected only during manual activation, in accordance with clinical observations disclosing that rigidity responds well to treatment. For tremor, it is believed that hyperactivity of inhibitory output from the globus pallidus may cause thalamic cell to oscillate, the indirect pathway in the present case, and that these oscillations 

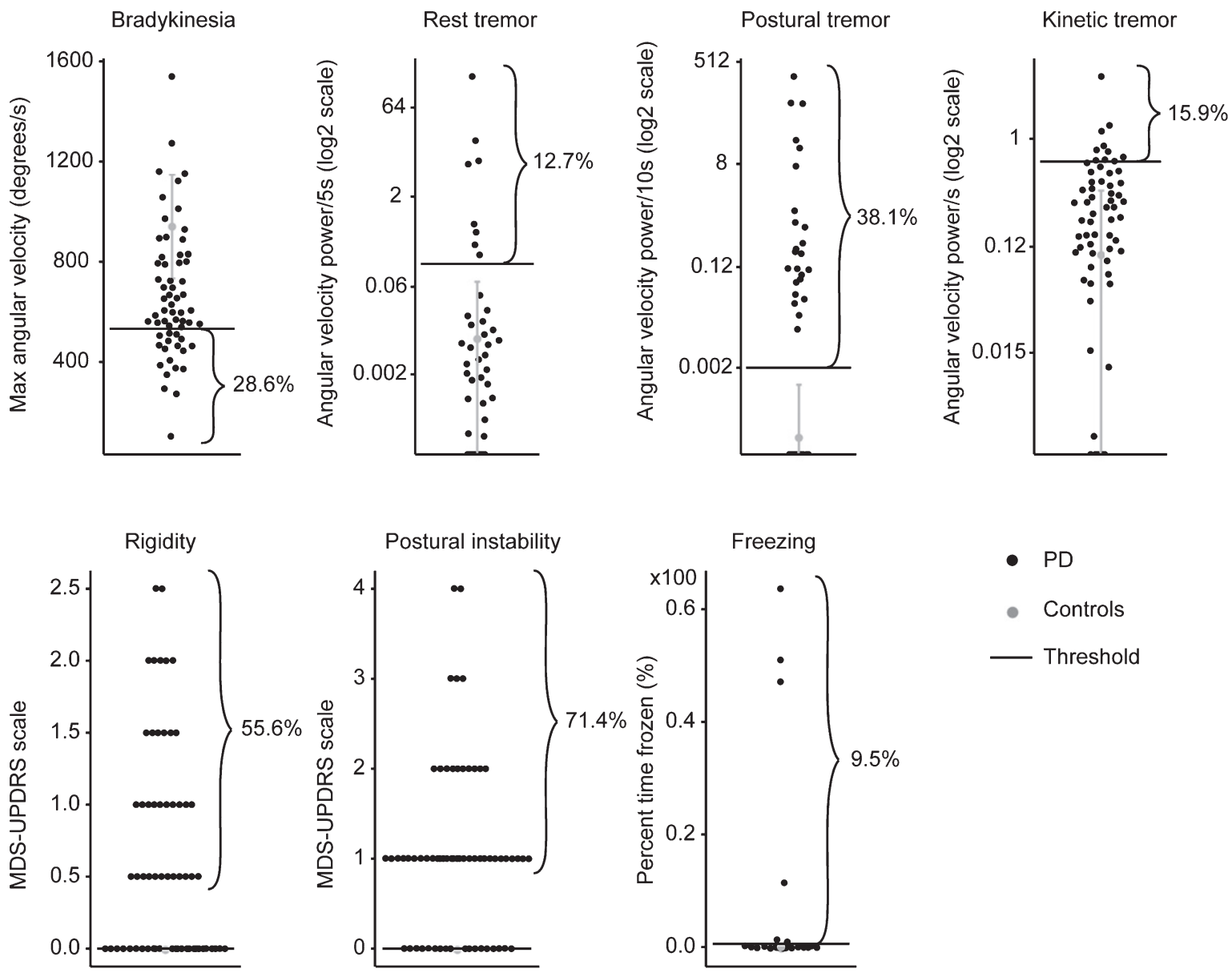

Fig. 3. Level of cardinal motor symptoms in the 63 patients with PD who presented with DID (black), compared to normative values obtained from the control group (grey). Mean plus two standard deviations of data from controls are Illustrated in gray. Individual data points from patients are illustrated in black.

could still be reinforced through the cerebellothalamo-cortical loop [46]. For postural instability and freezing of gait, the decreased cholinergic activity in the supplementary motor area, the parietal posterior cortex, the mesencephalic locomotor region, and the cerebellum may still be in cause [47], even with treatment.

Such dichotomy in the role of the different pathways in basal ganglia disorders is also supported by empirical evidence showing that, for example, patients with Huntington's chorea demonstrated no such bradykinesia while performing the same RAM task as performed by patients in the present study [48]. In Huntington's disease, it is the early preferential loss of D2 expressing GABAergic medium spiny striatal neurons associated with the indirect pathway that is proposed to be responsible for
Huntington's chorea [49], leaving the less affected direct pathway carrying the RAM command, at least early in the disease. Irrespective of the underlying mechanisms causing the simultaneous presence of DID and motor symptoms of PD, the neurophysiological bases responsible for causing the former should be regarded as distinct from those causing the latter.

Our results are not in agreement with the 'eitheror' pattern of motor response to levodopa over the time that is currently used to explain the state of patients $(O F F, O N$, or $O N$ with DID) [11, 12]. In fact, our results agree with clinical observations suggesting considerable overlap between symptoms. Accordingly, a more realistic illustration should include residual cardinal features of PD in the therapeutic window and, depending on the stage of the 


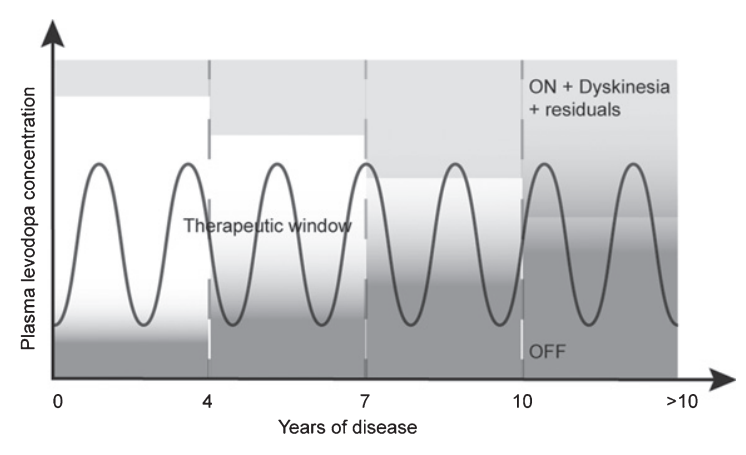

Fig. 4. Proposed pattern of motor response to levodopa over the time. This pattern suggests that some residual cardinal motor features of PD (i.e., bradykinesia, rigidity, postural instability and tremors) remain present in the therapeutic window, and could even overlap with dyskinesia.

disease, overlapping with peak-dose choreic-type DID (Fig. 4).

One important limitation of the current study is that we were not able to measure plasma levels of levodopa during the experiment to confirm the moment at which peak-dose was reached. However, this moment usually coincides with the peak-dose DID, during which we assessed the symptomatology of PD.

\section{Clinical considerations}

Our results also illustrate the harsh reality facing clinicians in trying to avoid DID while reducing the impact of cardinal features of PD. This scenario does not exist in the early stage of patients with PD because there is no need to adjust medication to avoid DID. If a drug dosage reduction is contemplated to reduce the levels of DID, a consequence could be an increase the underlying parkinsonism. If a change of medication from levodopa to dopamine agonists is contemplated, one should expect a reduction of anti-parkinsonian effect, as demonstrated by others $[3,4,50]$. In either case, patients must deal with both; the underlying PD motor symptoms and DID. Then, the tantalizing question would be; which of DID or parkinsonian motor symptoms cause the most problem for patients in their daily life activities? This question must be answered to identify the most relevant target(s) for treatment to maintain proper mobility for patients with advanced PD.

\section{ACKNOWLEDGMENTS}

We would like to thank the volunteers for their participation in the study, and the Canadian Institutes of Health Research (CIHR) who provided funding for the study (Grant number: 201303MOP-298400SDA-CFEB-56596).

\section{CONFLICT OF INTEREST}

The authors have no conflict of interest to report.

\section{REFERENCES}

[1] Gazewood JD, Richards DR, Clebak K (2013) Parkinson disease: An update. Am Fam Physician 87, 267-273.

[2] Sveinbjornsdottir S (2016) The clinical symptoms of Parkinson's disease. J Neurochem 139(Suppl 1), 318-324.

[3] Rascol O, Lozano A, Stern M, Poewe W (2011) Milestones in Parkinson's disease therapeutics. Mov Disord 26, 10721082.

[4] Fox SH, Katzenschlager R, Lim S-Y, Ravina B, Seppi K, Coelho M, Poewe W, Rascol O, Goetz CG, Sampaio C (2011) The Movement Disorder Society evidence-based medicine review update: Treatments for the motor symptoms of Parkinson's disease. Mov Disord 26 (Suppl 3), S2-41.

[5] Cenci M, Lindgren H (2007) Advances in understanding 1-DOPA-induced dyskinesia. Curr Opin Neurobiol 17, 665671.

[6] Cenci MA, Lundblad M (2006) Post- versus presynaptic plasticity in L-DOPA-induced dyskinesia. J Neurochem $\mathbf{9 9}$, 381-392.

[7] Bastide MF, Meissner WG, Picconi B, Fasano S, Fernagut P-O, Feyder M, Francardo V, Alcacer C, Ding Y, Brambilla R, Fisone G, Jon Stoessl A, Bourdenx M, Engeln M, Navailles S, De Deurwaerdére P, Ko WKD, Simola N, Morelli M, Groc L, Rodriguez M-C, Gurevich EV, Quik M, Morari M, Mellone M, Gardoni F, Tronci E, Guehl D, Tison F, Crossman AR, Kang UJ, Steece-Collier K, Fox S, Carta M, Angela Cenci M, Bézard E (2015) Pathophysiology of L-dopa-induced motor and non-motor complications in Parkinson's disease. Prog Neurobiol 132, 96-168.

[8] Rylander D, Parent M, O’Sullivan SS, Dovero S, Lees AJ, Bezard E, Descarries L, Cenci MA (2010) Maladaptive plasticity of serotonin axon terminals in levodopa-induced dyskinesia. Ann Neurol 68, 619-628.

[9] Guigoni C, Aubert I, Li Q, Gurevich VV, Benovic JL, Ferry S, Mach U, Stark H, Leriche L, Håkansson K, Bioulac BH, Gross CE, Sokoloff P, Fisone G, Gurevich EV, Bloch B, Bezard E (2005) Pathogenesis of levodopa-induced dyskinesia: Focus on D1 and D3 dopamine receptors. Parkinsonism Relat Disord 11(Suppl 1), S25-29.

[10] Fox SH (2013) Non-dopaminergic treatments for motor control in Parkinson's disease. Drugs 73, 1405-1415.

[11] Cenci MA (2014) Presynaptic mechanisms of 1-DOPAinduced dyskinesia: The findings, the debate, and the therapeutic implications. Front Neurol 5, 242.

[12] Jankovic J (2005) Motor fluctuations and dyskinesias in Parkinson's disease: Clinical manifestations. Mov Disord 20 (Suppl 11), S11-16.

[13] Ghassemi M, Lemieux S, Jog M, Edwards R, Duval C (2006) Bradykinesia in patients with Parkinson's disease having levodopa-induced dyskinesias. Brain Res Bull 69, 512-518. 
[14] Hallett M (2012) Parkinson's disease tremor: Pathophysiology. Parkinsonism Relat Disord 18(Suppl 1), S85-86.

[15] Bloem BR, Beckley DJ, van Dijk JG, Zwinderman AH, Remler MP, Roos RAC (1996) Influence of dopaminergic medication on automatic postural responses and balance impairment in Parkinson's disease. Mov Disord 11, 509-521.

[16] Luquin MR, Scipioni O, Vaamonde J, Gershanik O, Obeso JA (1992) Levodopa-induced dyskinesias in Parkinson's disease: Clinical and pharmacological classification. Mov Disord 7, 117-124.

[17] Metman LV, Del Dotto P, LePoole K, Konitsiotis S, Fang J, Chase TN (1999) Amantadine for levodopa-induced dyskinesias: A 1-year follow-up study. Arch Neurol 56, 1383-1386.

[18] Goetz CG, Damier P, Hicking C, Laska E, Müller T, Olanow CW, Rascol O, Russ H (2007) Sarizotan as a treatment for dyskinesias in Parkinson's disease: A double-blind placebocontrolled trial. Mov Disord 22, 179-186.

[19] Fox SH, Lang AE (2008) Levodopa-related motor complications-phenomenology. Mov Disord 23 (Suppl 3), S509-514.

[20] Bloem BR, Hausdorff JM, Visser JE, Giladi N (2004) Falls and freezing of gait in Parkinson's disease: A review of two interconnected, episodic phenomena. Mov Disord 19, 871-884.

[21] Hughes AJ, Daniel SE, Kilford L, Lees AJ (1992) Accuracy of clinical diagnosis of idiopathic Parkinson's disease: A clinico-pathological study of 100 cases. J Neurol Neurosurg Psychiatry 55, 181-184.

[22] Lebel K, Boissy P, Hamel M, Duval C (2013) Inertial measures of motion for clinical biomechanics: Comparative assessment of accuracy under controlled conditions - effect of velocity. PLoS One 8, e79945.

[23] Lebel K, Boissy P, Hamel M, Duval C (2015) Inertial measures of motion for clinical biomechanics: Comparative assessment of accuracy under controlled conditions changes in accuracy over time. PLoS One 10, e0118361.

[24] Lebel K, Boissy P, Nguyen H, Duval C (2016) Autonomous quality control of joint orientation measured with inertial sensors. Sensors 16, E1037.

[25] Ayachi FS, Nguyen HP, Lavigne-Pelletier C, Goubault E, Boissy P, Duval C (2016) Wavelet-based algorithm for autodetection of daily living activities of older adults captured by multiple inertial measurement units (IMUs). Physiol Meas 37, 442-461.

[26] Ayachi F, Nguyen H, Goubault E, Boissy P, Duval C (2016) The use of empirical mode decomposition-based algorithm and inertial measurement units to auto-detect daily living activities of healthy adults. IEEE Trans Neural Syst Rehabil Eng 24. doi: 10.1109/TNSRE.2016.2519413

[27] Nguyen HP, Ayachi F, Lavigne-Pelletier C, Blamoutier M, Rahimi F, Boissy P, Jog M, Duval C (2015) Auto detection and segmentation of physical activities during a Timed-Upand-Go (TUG) task in healthy older adults using multiple inertial sensors. J Neuroengineering Rehabil 12, 36.

[28] Nguyen H, Lebel K, Bogard S, Goubault E, Boissy P, Duval C (2018) Using inertial sensors to automatically detect and segment activities of daily living in people with Parkinson's disease. IEEE Trans Neural Syst Rehabil Eng 26, 197-204.

[29] Nguyen H, Lebel K, Boissy P, Bogard S, Goubault E, Duval C (2017) Auto detection and segmentation of daily living activities during a Timed Up and Go task in people with Parkinson's disease using multiple inertial sensors. $J \mathrm{Neu}$ roengineering Rehabil 14, 26.
[30] Beuter A, de Geoffroy A, Edwards R (1999) Analysis of rapid alternating movements in Cree subjects exposed to methylmercury and in subjects with neurological deficits. Environ Res 80, 64-79.

[31] Duval C, Panisset M, Sadikot AF (2001) The relationship between physiological tremor and the performance of rapid alternating movements in healthy elderly subjects. Exp Brain Res 139, 412-418.

[32] Okada M, Okada M (1983) A method for quantification of alternate pronation and supination of forearms. Comput Biomed Res Int J 16, 59-78.

[33] Duval C, Sadikot AF, Panisset M (2006) Bradykinesia in patients with essential tremor. Brain Res 1115, 213-216.

[34] Daneault J-F, Carignan B, Sadikot AF, Duval C (2013) Are quantitative and clinical measures of bradykinesia related in advanced Parkinson's disease? J Neurosci Methods 219, 220-223.

[35] Goubault E, Nguyen HP, Ayachi FS, Bogard S, Duval C (2017) Do bradykinesia and tremor interfere in voluntary movement of essential tremor patients? Preliminary findings. Tremor Other Hyperkinet Mov $(N Y)$ 7, 459 .

[36] Lemieux S, Ghassemi M, Jog M, Edwards R, Duval C (2007) The influence of levodopa-induced dyskinesias on manual tracking in patients with Parkinson's disease. Exp Brain Res 176, 465-475.

[37] Moore ST, Yungher DA, Morris TR, Dilda V, MacDougall HG, Shine JM, Naismith SL, Lewis SJG (2013) Autonomous identification of freezing of gait in Parkinson's disease from lower-body segmental accelerometry. J Neuroengineering Rehabil 10, 19.

[38] Aquino CC, Fox SH (2015) Clinical spectrum of levodopainduced complications. Mov Disord 30, 80-89.

[39] Duval C, Fenney A, Jog MS (2009) The dynamic relationship between voluntary and involuntary motor behaviours in patients with basal ganglia disorders. In The Basal Ganglia IX, Advances in Behavioral Biology 58 H.J. Groenewegen et al. (eds.) Springer Science+Business Media, LLC 2009.

[40] Cenci MA, Ohlin KE, Rylander D (2009) Plastic effects of L-DOPA treatment in the basal ganglia and their relevance to the development of dyskinesia. Parkinsonism Relat Disord 15 (Supplement 3), S59-S63.

[41] Little S, Pogosyan A, Kuhn AA, Brown P (2012) $\beta$ band stability over time correlates with Parkinsonian rigidity and bradykinesia. Exp Neurol 236, 383-388.

[42] Weinberger M, Hutchison WD, Dostrovsky JO (2009) Pathological subthalamic nucleus oscillations in PD: Can they be the cause of bradykinesia and akinesia? Exp Neurol 219, 58-61.

[43] Meara RJ, Cody FW (1992) Relationship between electromyographic activity and clinically assessed rigidity studied at the wrist joint in Parkinson's disease. Brain 115( $\mathrm{Pt}$ 4), 1167-1180.

[44] Rothwell J, Obeso J, Traub M, Marsden C (1983) The behaviour of the long-latency stretch reflex in patients with Parkinson's disease. J Neurol Neurosurg Psychiatry 46, 35-44.

[45] Baradaran N, Tan SN, Liu A, Ashoori A, Palmer SJ, Wang ZJ, Oishi MMK, McKeown MJ (2013) Parkinson's disease rigidity: Relation to brain connectivity and motor performance. Front Neurol 4, 67.

[46] Duval C, Daneault J-F, Hutchison WD, Sadikot AF (2016) A brain network model explaining tremor in Parkinson's disease. Neurobiol Dis 85, 49-59. 
[47] Collomb-Clerc A, Welter M-L (2015) Effects of deep brain stimulation on balance and gait in patients with Parkinson's disease: A systematic neurophysiological review. Neurophysiol Clin 45, 371-388.

[48] Fenney A, Jog MS, Duval C (2008) Bradykinesia is not a "systematic" feature of adult-onset Huntington's disease; implications for basal ganglia pathophysiology. Brain Res 1193, 67-75.
[49] Reiner A, Albin RL, Anderson KD, D'Amato CJ, Penney JB, Young AB (1988) Differential loss of striatal projection neurons in Huntington disease. Proc Natl Acad Sci U S A 85, 5733-5737.

[50] Jann MW (2011) Advanced strategies for treatment of Parkinson's disease: The role of early treatment. Am J Manag Care 17 (Suppl 12), S315-321. 